\title{
Anharmonic Vibrational Analyses of Pentapeptide Conformations Explored with Enhanced Sampling Simulations
}

\section{Supporting Information}

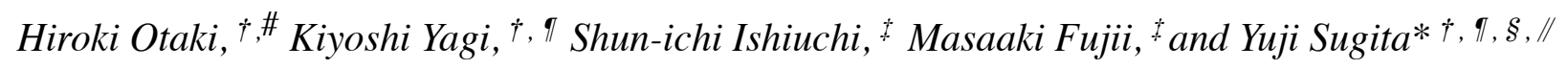

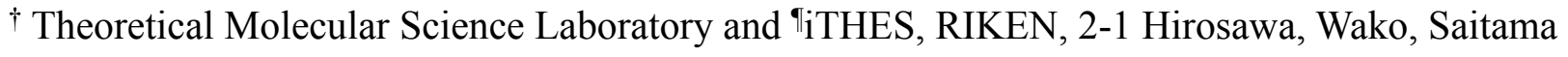
351-0198, Japan.

* Laboratory for Chemistry and Life Science, Institute for Innovative Research, Tokyo Institute of Technology, 4259 Nagatsuta-cho, Midori-ku, Yokohama 226-8503, Japan.

$\S$ RIKEN Advanced Institute for Computational Science, 7-1-26 Minatojima-Minamimachi, Chuo-ku, Kobe, Hyogo 650-0047, Japan.

/RIKEN Quantitative Biology Center, 1-6-5 Minatojima-Minamimachi, Chuo-ku, Kobe, Hyogo 650-0047, Japan. 


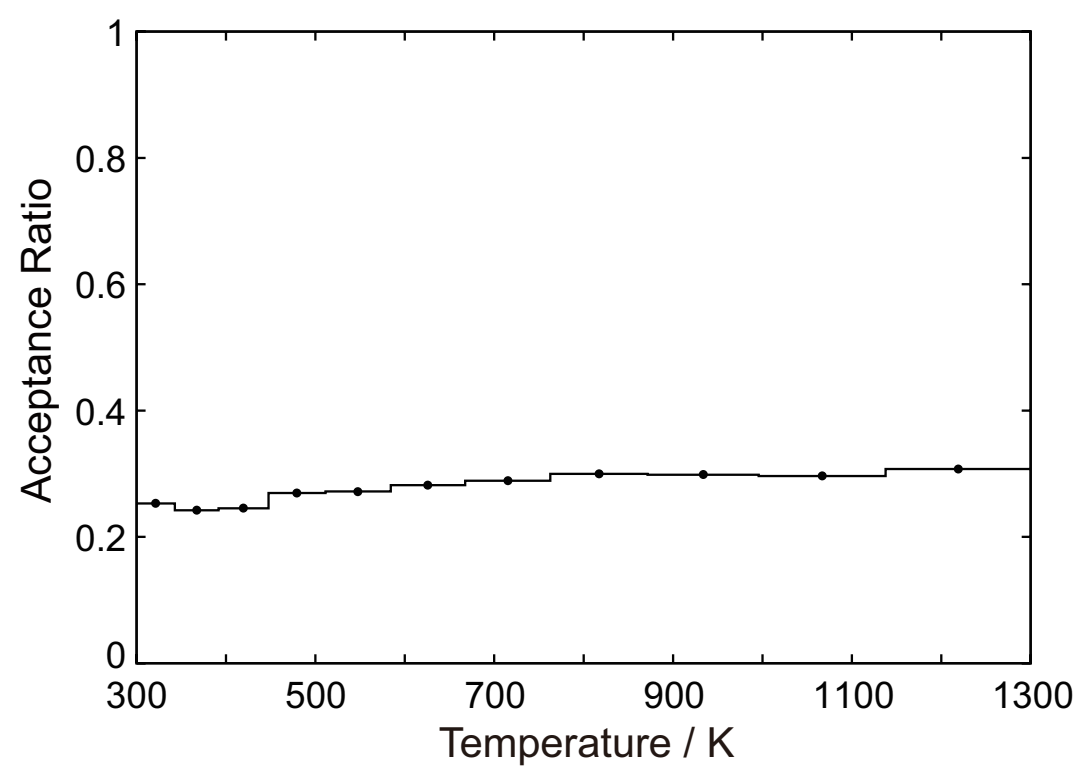

Figure S1. Acceptance ratio of adjacent replicas.

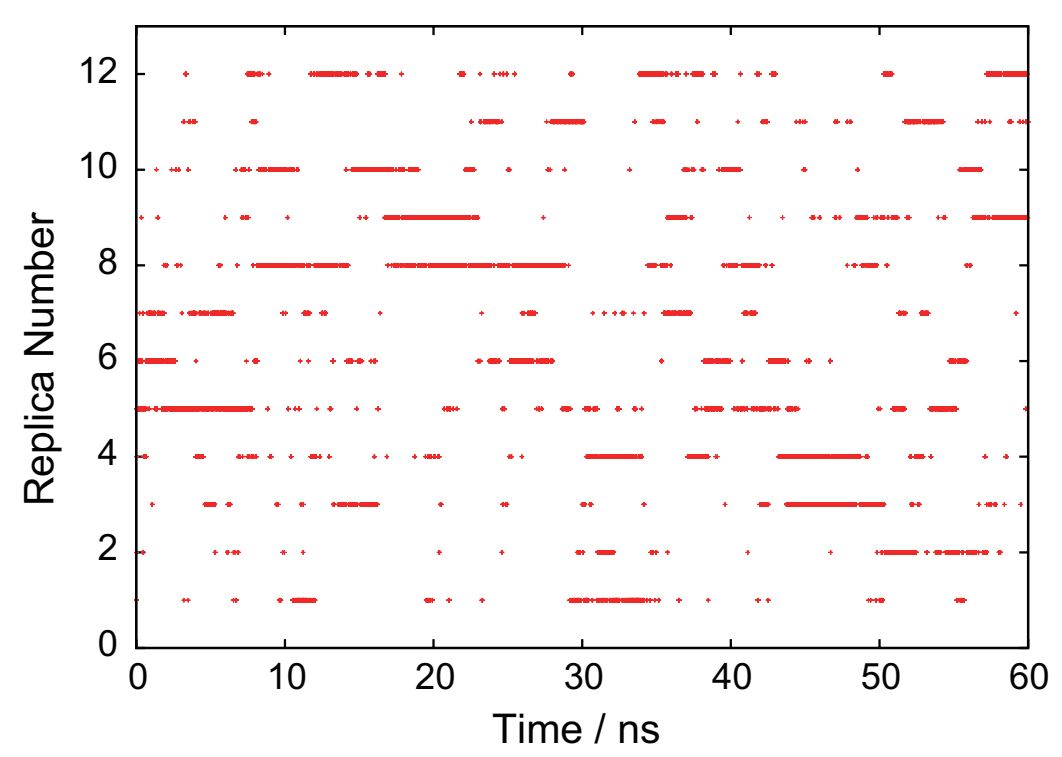

Figure S2. Replica number of the trajectory at 300 $\mathrm{K}$ as a function of time. 

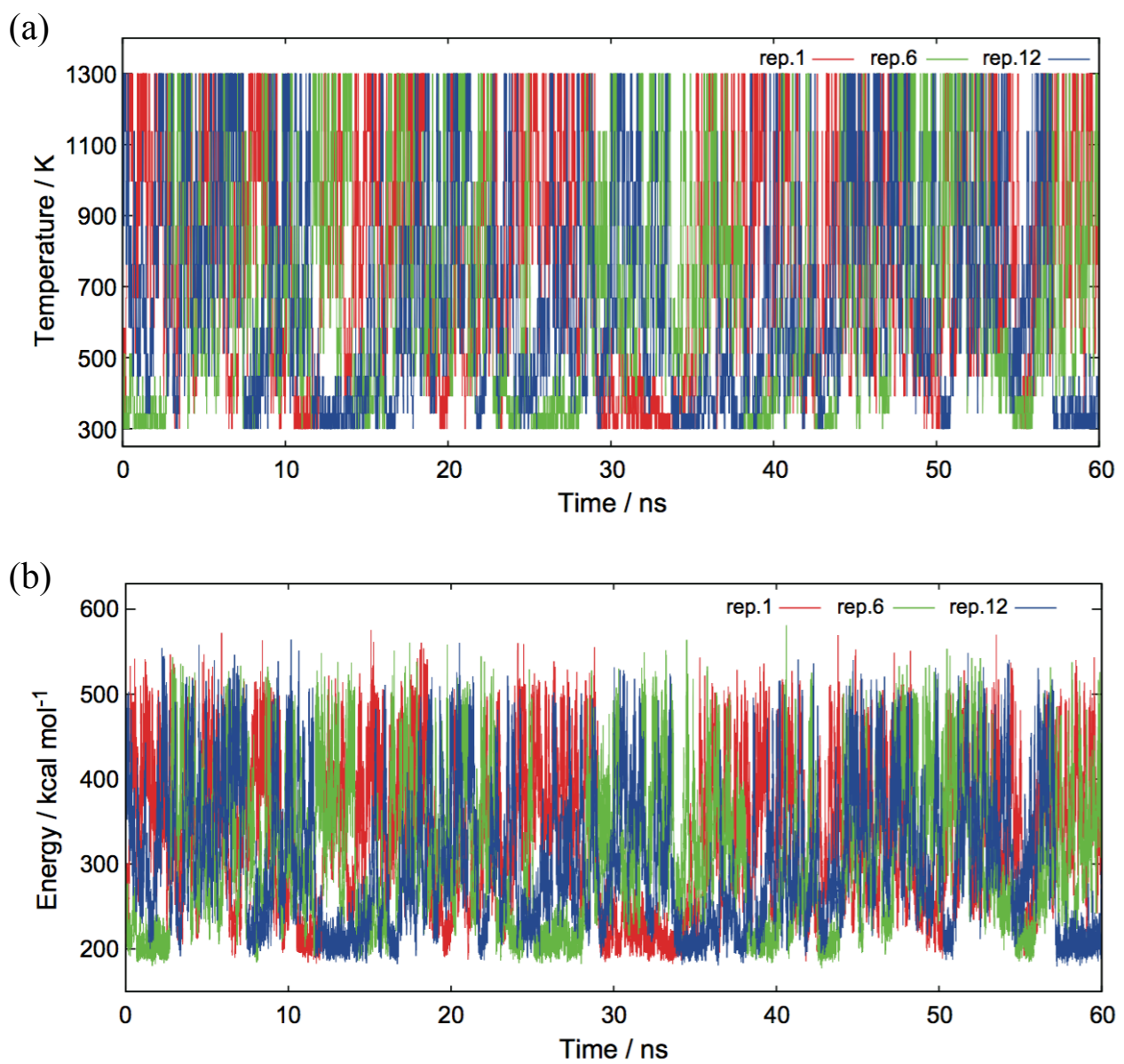

Figure S3. (a) Temperature and (b) potential energy of a replica 1,6 , and 12 as a function of time. 


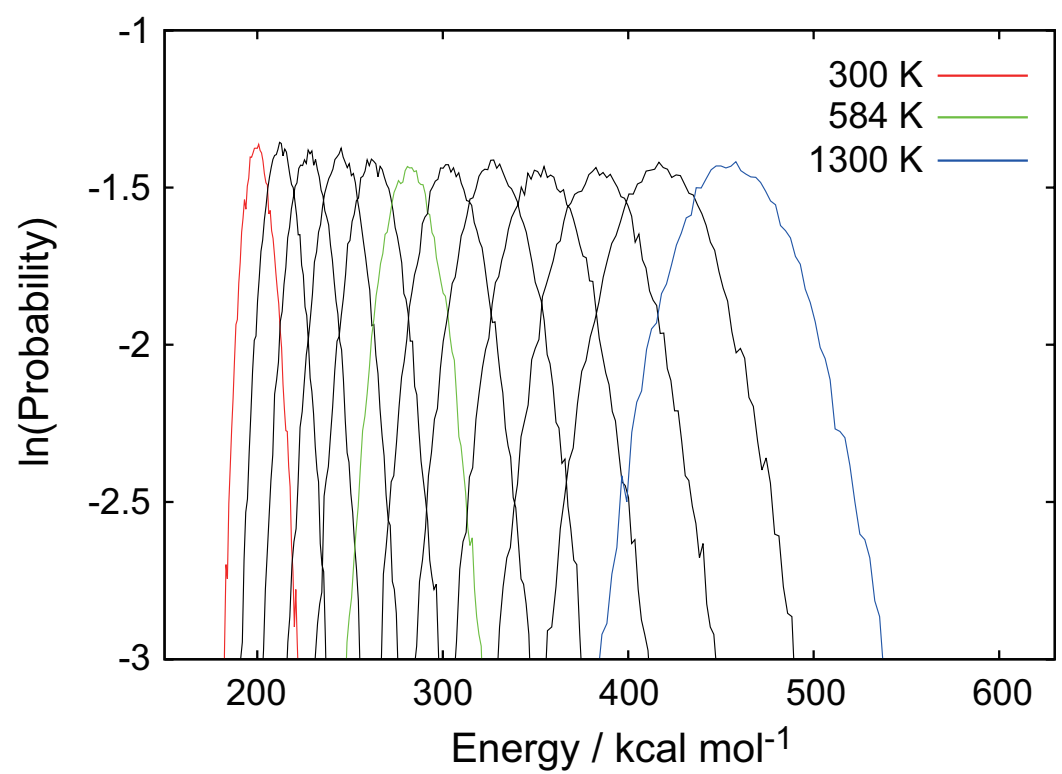

Figure S4. Canonical probability distribution of the potential energy with different temperature. 


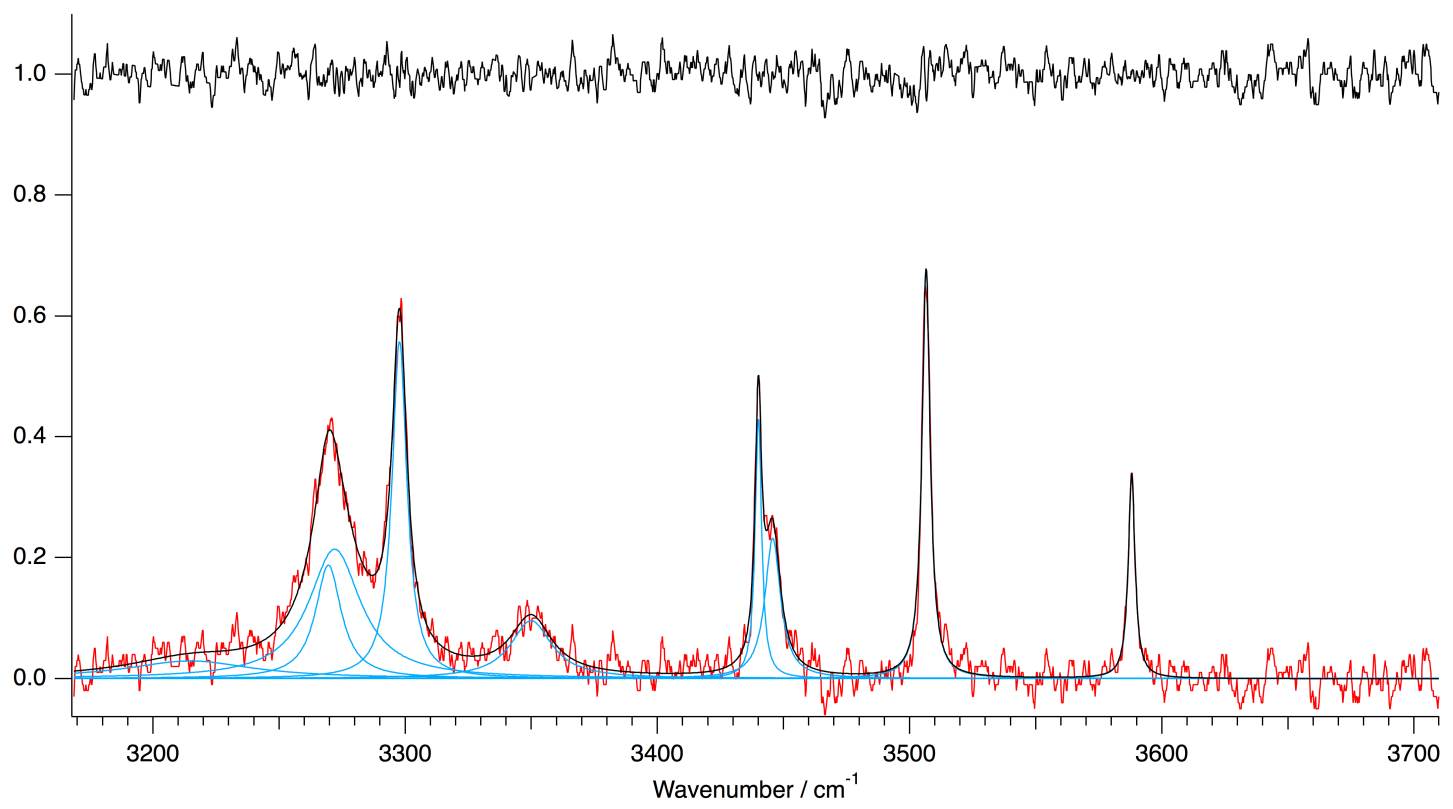

Figure S5. Fitting of the observed spectrum (red) to 9 Lorentz functions (black). The components of the Lorentz functions are shown in blue. 
Table S1. Character of Vibrational Modes Selected in the Vibrational Structure Calculations of 30-, 40-, 60-, and 80-Mode Models

\begin{tabular}{|c|c|c|c|c|c|c|c|c|c|c|c|c|c|c|c|}
\hline \multirow[t]{2}{*}{ mode } & \multirow[t]{2}{*}{ omega } & \multirow[t]{2}{*}{ character } & \multicolumn{4}{|c|}{ model } & \multicolumn{9}{|c|}{ weight } \\
\hline & & & 30 & 40 & 60 & 80 & $\mathrm{NH}_{\mathrm{I}}$ & $\mathrm{NH}_{\mathrm{V}}$ & $\mathrm{NH}_{\mathrm{S} 4}$ & $\mathrm{NH}_{\mathrm{F}}$ & $\left(\mathrm{NH}_{2}\right)_{\mathrm{S} 1}$ & $\left(\mathrm{NH}_{2}\right)_{\mathrm{F}}$ & $\mathrm{OH}_{\mathrm{S} 1}$ & $\mathrm{OH}_{\mathrm{S} 4}$ & Other \\
\hline 7 & 55.0 & - & & & & ○ & 0.00 & 0.00 & 0.01 & 0.00 & 0.09 & 0.05 & 0.07 & 0.01 & 0.76 \\
\hline 9 & 65.2 & - & & & $\circ$ & ○ & 0.01 & 0.01 & 0.00 & 0.01 & 0.01 & 0.02 & 0.11 & 0.00 & 0.82 \\
\hline 10 & 66.2 & - & & & ○ & ○ & 0.00 & 0.00 & 0.00 & 0.01 & 0.00 & 0.01 & 0.00 & 0.20 & 0.76 \\
\hline 13 & 76.0 & - & & & & ○ & 0.00 & 0.01 & 0.02 & 0.02 & 0.00 & 0.02 & 0.03 & 0.09 & 0.81 \\
\hline 17 & 101.9 & - & & & & o & 0.01 & 0.00 & 0.01 & 0.00 & 0.05 & 0.02 & 0.03 & 0.11 & 0.76 \\
\hline 19 & 118.5 & - & & $\circ$ & $\circ$ & ○ & 0.03 & 0.03 & 0.01 & 0.01 & 0.02 & 0.13 & 0.09 & 0.03 & 0.67 \\
\hline 20 & 120.3 & - & & & $\circ$ & ○ & 0.03 & 0.01 & 0.01 & 0.02 & 0.02 & 0.03 & 0.02 & 0.17 & 0.68 \\
\hline 25 & 167.9 & - & & & & ○ & 0.01 & 0.01 & 0.16 & 0.16 & 0.01 & 0.02 & 0.01 & 0.02 & 0.61 \\
\hline 30 & 206.7 & - & & & & $\circ$ & 0.01 & 0.01 & 0.00 & 0.00 & 0.09 & 0.02 & 0.29 & 0.00 & 0.58 \\
\hline 31 & 206.7 & - & $\circ$ & $\circ$ & $\circ$ & ○ & 0.05 & 0.07 & 0.00 & 0.01 & 0.13 & 0.00 & 0.00 & 0.01 & 0.72 \\
\hline 32 & 227.5 & - & & & ○ & ○ & 0.02 & 0.02 & 0.00 & 0.00 & 0.06 & 0.00 & 0.00 & 0.00 & 0.89 \\
\hline 34 & 241.4 & - & & & & o & 0.06 & 0.02 & 0.00 & 0.01 & 0.02 & 0.00 & 0.01 & 0.00 & 0.89 \\
\hline 35 & 249.7 & - & & & $\circ$ & ○ & 0.00 & 0.00 & 0.00 & 0.08 & 0.03 & 0.03 & 0.00 & 0.00 & 0.85 \\
\hline 36 & 250.4 & - & & & $\circ$ & ○ & 0.06 & 0.01 & 0.02 & 0.02 & 0.08 & 0.01 & 0.01 & 0.03 & 0.76 \\
\hline 37 & 259.1 & $\begin{array}{c}\left(\mathrm{NH}_{2}\right)_{\mathrm{S}^{-}} \\
\quad \text { wag }\end{array}$ & $\circ$ & 0 & ○ & ○ & 0.02 & 0.01 & 0.02 & 0.01 & 0.42 & 0.01 & 0.01 & 0.09 & 0.43 \\
\hline 38 & 262.6 & $\begin{array}{l}\left(\mathrm{NH}_{2}\right)_{\mathrm{S1}^{-}} \\
\quad \text { wag }\end{array}$ & $\circ$ & 0 & 0 & ○ & 0.00 & 0.01 & 0.10 & 0.00 & 0.21 & 0.00 & 0.01 & 0.22 & 0.45 \\
\hline 39 & 271.3 & - & & ○ & O & O & 0.00 & 0.00 & 0.00 & 0.00 & 0.04 & 0.00 & 0.00 & 0.00 & 0.95 \\
\hline 40 & 282.2 & $\begin{array}{c}\left(\mathrm{NH}_{2}\right)_{\mathrm{S}^{-}} \\
\quad \text { wag }\end{array}$ & ○ & ○ & ○ & O & 0.01 & 0.01 & 0.03 & 0.03 & 0.14 & 0.02 & 0.00 & 0.01 & 0.76 \\
\hline 41 & 290.6 & - & & & $\circ$ & 0 & 0.01 & 0.02 & 0.03 & 0.05 & 0.05 & 0.02 & 0.00 & 0.02 & 0.80 \\
\hline 45 & 319.7 & - & & & & ○ & 0.07 & 0.01 & 0.09 & 0.05 & 0.03 & 0.10 & 0.02 & 0.01 & 0.62 \\
\hline
\end{tabular}




\begin{tabular}{|c|c|c|c|c|c|c|c|c|c|c|c|c|c|c|c|}
\hline \multirow[t]{2}{*}{ mode } & \multirow[t]{2}{*}{ omega } & \multirow[t]{2}{*}{ character } & \multicolumn{4}{|c|}{ model } & \multicolumn{9}{|c|}{ weight } \\
\hline & & & 30 & 40 & 60 & 80 & $\mathrm{NH}_{\mathrm{I}}$ & $\mathrm{NH}_{\mathrm{V}}$ & $\mathrm{NH}_{\mathrm{S} 4}$ & $\mathrm{NH}_{\mathrm{F}}$ & $\left(\mathrm{NH}_{2}\right)_{\mathrm{S} 1}$ & $\left(\mathrm{NH}_{2}\right)_{\mathrm{F}}$ & $\mathrm{OH}_{\mathrm{S} 1}$ & $\mathrm{OH}_{\mathrm{S} 4}$ & Other \\
\hline 46 & 336.1 & - & & & ○ & ○ & 0.01 & 0.09 & 0.01 & 0.07 & 0.19 & 0.03 & 0.01 & 0.01 & 0.58 \\
\hline 49 & 356.9 & - & & & & 0 & 0.01 & 0.14 & 0.11 & 0.09 & 0.01 & 0.03 & 0.01 & 0.01 & 0.60 \\
\hline 51 & 387.1 & - & & & & 0 & 0.17 & 0.08 & 0.01 & 0.00 & 0.05 & 0.00 & 0.03 & 0.00 & 0.66 \\
\hline 53 & 418.6 & - & & & ○ & 0 & 0.00 & 0.00 & 0.00 & 0.00 & 0.00 & 0.00 & 0.00 & 0.01 & 0.99 \\
\hline 54 & 430.3 & $\mathrm{OH}_{\mathrm{S} 4-\mathrm{oop}}$ & ○ & O & ○ & 0 & 0.00 & 0.00 & 0.04 & 0.01 & 0.00 & 0.00 & 0.00 & 0.90 & 0.06 \\
\hline 56 & 460.6 & - & & O & ○ & 0 & 0.05 & 0.03 & 0.03 & 0.01 & 0.07 & 0.03 & 0.12 & 0.00 & 0.66 \\
\hline 57 & 465.1 & - & & & & 0 & 0.03 & 0.01 & 0.01 & 0.02 & 0.04 & 0.07 & 0.06 & 0.00 & 0.77 \\
\hline 59 & 486.6 & - & & & & 0 & 0.00 & 0.01 & 0.06 & 0.04 & 0.00 & 0.01 & 0.00 & 0.08 & 0.80 \\
\hline 60 & 503.9 & - & O & O & ○ & 0 & 0.05 & 0.02 & 0.00 & 0.00 & 0.18 & 0.00 & 0.14 & 0.00 & 0.60 \\
\hline 61 & 538.7 & - & & ० & ○ & 0 & 0.01 & 0.10 & 0.05 & 0.01 & 0.00 & 0.01 & 0.05 & 0.01 & 0.76 \\
\hline 63 & 554.0 & - & ० & ० & ○ & 0 & 0.06 & 0.07 & 0.03 & 0.00 & 0.01 & 0.01 & 0.08 & 0.00 & 0.74 \\
\hline 64 & 557.2 & $\mathrm{OH}_{\mathrm{S1}-\mathrm{oop}}$ & ○ & ○ & ○ & 0 & 0.02 & 0.01 & 0.00 & 0.00 & 0.06 & 0.04 & 0.72 & 0.00 & 0.15 \\
\hline 67 & 605.4 & $\left(\mathrm{NH}_{2}\right)_{\mathrm{F}}-\mathrm{wag}$ & O & O & ○ & 0 & 0.00 & 0.01 & 0.02 & 0.07 & 0.00 & 0.59 & 0.02 & 0.02 & 0.26 \\
\hline 68 & 610.8 & $\mathrm{NH}_{\mathrm{S} 4-\mathrm{oop}}$ & O & O & ○ & 0 & 0.00 & 0.07 & 0.40 & 0.04 & 0.00 & 0.08 & 0.00 & 0.01 & 0.39 \\
\hline 69 & 632.9 & - & & O & ○ & 0 & 0.00 & 0.00 & 0.10 & 0.00 & 0.00 & 0.08 & 0.00 & 0.01 & 0.80 \\
\hline 70 & 635.7 & - & & & ○ & 0 & 0.00 & 0.00 & 0.09 & 0.00 & 0.00 & 0.08 & 0.00 & 0.01 & 0.82 \\
\hline 71 & 661.7 & - & O & O & ○ & 0 & 0.00 & 0.01 & 0.15 & 0.02 & 0.00 & 0.14 & 0.00 & 0.02 & 0.66 \\
\hline 72 & 679.0 & - & & & ○ & $\circ$ & 0.05 & 0.14 & 0.05 & 0.02 & 0.03 & 0.02 & 0.00 & 0.00 & 0.67 \\
\hline 73 & 681.9 & - & & & & 0 & 0.11 & 0.09 & 0.07 & 0.05 & 0.04 & 0.00 & 0.00 & 0.00 & 0.64 \\
\hline 74 & 682.7 & $\begin{array}{c}\mathrm{NH}_{\mathrm{F}}, \\
\left(\mathrm{NH}_{2}\right)_{\mathrm{F}} \text {-oop }\end{array}$ & & 0 & ○ & 0 & 0.04 & 0.15 & 0.05 & 0.17 & 0.00 & 0.03 & 0.00 & 0.00 & 0.56 \\
\hline 75 & 701.7 & $\left(\mathrm{NH}_{2}\right)_{\mathrm{F}}$-oop & O & O & $\circ$ & 0 & 0.01 & 0.01 & 0.02 & 0.00 & 0.00 & 0.89 & 0.00 & 0.00 & 0.07 \\
\hline 77 & 731.2 & $\begin{array}{c}\mathrm{NH}_{\mathrm{I}}, \mathrm{NH}_{\mathrm{V}} \\
\mathrm{NH}_{\mathrm{F}} \text {-oop }\end{array}$ & & ○ & ○ & 0 & 0.16 & 0.16 & 0.05 & 0.19 & 0.02 & 0.00 & 0.00 & 0.01 & 0.40 \\
\hline
\end{tabular}




\begin{tabular}{|c|c|c|c|c|c|c|c|c|c|c|c|c|c|c|c|}
\hline \multirow[t]{2}{*}{ mode } & \multirow[t]{2}{*}{ omega } & \multirow[t]{2}{*}{ character } & \multicolumn{4}{|c|}{ model } & \multicolumn{9}{|c|}{ weight } \\
\hline & & & 30 & 40 & 60 & 80 & $\mathrm{NH}_{\mathrm{I}}$ & $\mathrm{NH}_{\mathrm{V}}$ & $\mathrm{NH}_{\mathrm{S} 4}$ & $\mathrm{NH}_{\mathrm{F}}$ & $\left(\mathrm{NH}_{2}\right)_{\mathrm{S} 1}$ & $\left(\mathrm{NH}_{2}\right)_{\mathrm{F}}$ & $\mathrm{OH}_{\mathrm{S} 1}$ & $\mathrm{OH}_{\mathrm{S} 4}$ & Other \\
\hline 78 & 748.4 & $\begin{array}{c}\mathrm{NH}_{\mathrm{I}}, \mathrm{NH}_{\mathrm{V}}, \\
\mathrm{NH}_{\mathrm{F}} \text {-oop }\end{array}$ & O & O & O & O & 0.12 & 0.43 & 0.04 & 0.10 & 0.00 & 0.00 & 0.00 & 0.01 & 0.29 \\
\hline 79 & 751.8 & $\mathrm{NH}_{\mathrm{I}}$-oop & O & o & o & ○ & 0.23 & 0.05 & 0.04 & 0.25 & 0.02 & 0.02 & 0.01 & 0.01 & 0.37 \\
\hline 82 & 781.1 & - & & & & O & 0.09 & 0.06 & 0.01 & 0.01 & 0.00 & 0.03 & 0.00 & 0.00 & 0.80 \\
\hline 84 & 799.1 & - & & & o & $\circ$ & 0.13 & 0.04 & 0.03 & 0.05 & 0.02 & 0.03 & 0.00 & 0.01 & 0.70 \\
\hline 85 & 804.8 & $\mathrm{NH}_{\mathrm{I}}$-oop & & & O & ○ & 0.20 & 0.02 & 0.02 & 0.04 & 0.01 & 0.03 & 0.00 & 0.01 & 0.66 \\
\hline 91 & 879.8 & - & & & & ○ & 0.00 & 0.00 & 0.01 & 0.06 & 0.08 & 0.00 & 0.00 & 0.05 & 0.79 \\
\hline 92 & 880.8 & $\begin{array}{c}\left(\mathrm{NH}_{2}\right)_{\mathrm{S}^{-}} \\
\text {oop }\end{array}$ & ○ & ○ & ० & O & 0.00 & 0.00 & 0.00 & 0.01 & 0.74 & 0.00 & 0.00 & 0.01 & 0.24 \\
\hline 104 & 987.4 & - & & & & ○ & 0.04 & 0.02 & 0.00 & 0.00 & 0.17 & 0.00 & 0.03 & 0.00 & 0.73 \\
\hline 109 & 1014.1 & - & & & & O & 0.00 & 0.02 & 0.08 & 0.06 & 0.00 & 0.00 & 0.00 & 0.12 & 0.73 \\
\hline 125 & 1158.4 & - & & $\circ$ & ○ & $\circ$ & 0.00 & 0.00 & 0.00 & 0.01 & 0.00 & 0.65 & 0.00 & 0.00 & 0.35 \\
\hline 128 & 1182.4 & - & & & ○ & ○ & 0.01 & 0.00 & 0.00 & 0.00 & 0.17 & 0.00 & 0.15 & 0.00 & 0.67 \\
\hline 133 & 1213.1 & - & & & & ○ & 0.00 & 0.00 & 0.02 & 0.14 & 0.00 & 0.03 & 0.00 & 0.06 & 0.74 \\
\hline 134 & 1221.2 & - & & & O & ○ & 0.00 & 0.00 & 0.03 & 0.04 & 0.00 & 0.03 & 0.00 & 0.13 & 0.77 \\
\hline 137 & 1246.2 & $\mathrm{C}_{\mathrm{V}} \mathrm{N}_{\mathrm{S} 4}-\mathrm{Str}$ & & & ○ & $\circ$ & 0.00 & 0.09 & 0.23 & 0.00 & 0.00 & 0.00 & 0.00 & 0.00 & 0.67 \\
\hline 139 & 1276.5 & $\mathrm{C}_{\mathrm{S} 1} \mathrm{~N}_{\mathrm{I}}-\mathrm{Str}$ & & & & ○ & 0.23 & 0.02 & 0.00 & 0.00 & 0.07 & 0.00 & 0.03 & 0.00 & 0.64 \\
\hline 140 & 1287.9 & $\mathrm{C}_{\mathrm{S} 4} \mathrm{~N}_{\mathrm{F}}-\mathrm{Str}$ & & & & $\circ$ & 0.00 & 0.00 & 0.00 & 0.16 & 0.00 & 0.08 & 0.00 & 0.01 & 0.74 \\
\hline 156 & 1380.4 & $\begin{array}{c}\mathrm{OH}-\mathrm{b}+ \\
\mathrm{CH}_{2}-\mathrm{b}\end{array}$ & & & & $\circ$ & 0.00 & 0.00 & 0.00 & 0.00 & 0.09 & 0.00 & 0.15 & 0.01 & 0.75 \\
\hline 157 & 1381.7 & $\begin{array}{c}\mathrm{OH}-\mathrm{b}+ \\
\mathrm{CH}_{2}-\mathrm{b}\end{array}$ & & & o & ○ & 0.00 & 0.00 & 0.00 & 0.00 & 0.00 & 0.00 & 0.01 & 0.19 & 0.80 \\
\hline 161 & 1406.7 & $\begin{array}{c}\mathrm{OH}-\mathrm{b}+ \\
\mathrm{CH}_{2}-\mathrm{b}\end{array}$ & & $\circ$ & ○ & O & 0.00 & 0.00 & 0.00 & 0.00 & 0.01 & 0.00 & 0.31 & 0.05 & 0.63 \\
\hline
\end{tabular}




\begin{tabular}{|c|c|c|c|c|c|c|c|c|c|c|c|c|c|c|c|}
\hline \multirow[t]{2}{*}{ mode } & \multirow[t]{2}{*}{ omega } & \multirow[t]{2}{*}{ character } & \multicolumn{4}{|c|}{ model } & \multicolumn{9}{|c|}{ weight } \\
\hline & & & 30 & 40 & 60 & 80 & $\mathrm{NH}_{\mathrm{I}}$ & $\mathrm{NH}_{\mathrm{V}}$ & $\mathrm{NH}_{\mathrm{S} 4}$ & $\mathrm{NH}_{\mathrm{F}}$ & $\left(\mathrm{NH}_{2}\right)_{\mathrm{S} 1}$ & $\left(\mathrm{NH}_{2}\right)_{\mathrm{F}}$ & $\mathrm{OH}_{\mathrm{S} 1}$ & $\mathrm{OH}_{\mathrm{S} 4}$ & Other \\
\hline 162 & 1407.4 & $\begin{array}{c}\mathrm{OH}-\mathrm{b}+ \\
\mathrm{CH}_{2}-\mathrm{b}\end{array}$ & & & O & O & 0.00 & 0.00 & 0.00 & 0.01 & 0.01 & 0.00 & 0.17 & 0.09 & 0.72 \\
\hline 183 & 1539.7 & $\mathrm{NH}_{\mathrm{S} 4}-\mathrm{b}$ & O & O & O & ○ & 0.00 & 0.00 & 0.63 & 0.06 & 0.00 & 0.00 & 0.00 & 0.00 & 0.31 \\
\hline 184 & 1565.2 & $\mathrm{NH}_{\mathrm{I}}-\mathrm{b}$ & o & ○ & o & o & 0.50 & 0.01 & 0.03 & 0.20 & 0.00 & 0.00 & 0.00 & 0.00 & 0.26 \\
\hline 185 & 1568.5 & $\mathrm{NH}_{\mathrm{F}}-\mathrm{b}$ & & ○ & ○ & o & 0.23 & 0.00 & 0.04 & 0.44 & 0.00 & 0.00 & 0.00 & 0.00 & 0.28 \\
\hline 186 & 1591.1 & $\mathrm{NH}_{\mathrm{V}}-\mathrm{b}$ & o & o & o & $\circ$ & 0.00 & 0.78 & 0.00 & 0.01 & 0.00 & 0.00 & 0.00 & 0.00 & 0.21 \\
\hline 189 & 1662.1 & $\left(\mathrm{NH}_{2}\right)_{\mathrm{F}}-\mathrm{b}$ & 0 & O & O & 0 & 0.00 & 0.00 & 0.00 & 0.00 & 0.00 & 0.83 & 0.00 & 0.00 & 0.17 \\
\hline 190 & 1670.1 & $\left(\mathrm{NH}_{2}\right)_{\mathrm{S} 1}-\mathrm{b}$ & O & ० & ○ & O & 0.00 & 0.00 & 0.00 & 0.00 & 0.94 & 0.00 & 0.00 & 0.00 & 0.06 \\
\hline 139 & 1276.5 & $\mathrm{C}_{\mathrm{S} 1} \mathrm{~N}_{\mathrm{I}}-\mathrm{str}$ & & & & $\circ$ & 0.23 & 0.02 & 0.00 & 0.00 & 0.07 & 0.00 & 0.03 & 0.00 & 0.64 \\
\hline 195 & 1738.1 & $\mathrm{CO}_{\mathrm{F}}$ & & & 0 & o & 0.00 & 0.00 & 0.00 & 0.00 & 0.00 & 0.13 & 0.00 & 0.00 & 0.86 \\
\hline 228 & 3427.8 & $\mathrm{NH}_{\mathrm{I}}-\mathrm{str}$ & ○ & ० & ○ & $\circ$ & 0.96 & 0.02 & 0.00 & 0.01 & 0.00 & 0.01 & 0.00 & 0.00 & 0.00 \\
\hline 229 & 3458.7 & $\mathrm{NH}_{\mathrm{V}^{-}} \mathrm{str}$ & ○ & ० & ○ & $\circ$ & 0.03 & 0.93 & 0.00 & 0.04 & 0.00 & 0.00 & 0.00 & 0.00 & 0.00 \\
\hline 230 & 3475.0 & $\mathrm{NH}_{\mathrm{F}}-\mathrm{str}$ & ○ & O & ० & $\circ$ & 0.00 & 0.05 & 0.00 & 0.95 & 0.00 & 0.00 & 0.00 & 0.00 & 0.00 \\
\hline 231 & 3498.1 & $\begin{array}{c}\text { sym- } \\
\left(\mathrm{NH}_{2}\right)_{\mathrm{S} 1}\end{array}$ & O & O & O & O & 0.00 & 0.00 & 0.00 & 0.00 & 1.00 & 0.00 & 0.00 & 0.00 & 0.00 \\
\hline 232 & 3498.9 & sym- $\left(\mathrm{NH}_{2}\right)_{\mathrm{F}}$ & ○ & ○ & ○ & ○ & 0.01 & 0.00 & 0.00 & 0.00 & 0.00 & 0.99 & 0.00 & 0.00 & 0.00 \\
\hline 233 & 3587.0 & $\begin{array}{l}\text { asym- } \\
\left(\mathrm{NH}_{2}\right)_{\mathrm{S} 1}\end{array}$ & O & O & O & ० & 0.00 & 0.00 & 0.00 & 0.00 & 1.00 & 0.00 & 0.00 & 0.00 & 0.00 \\
\hline 234 & 3604.7 & $\mathrm{NH}_{\mathrm{S} 4-\mathrm{str}}$ & ○ & O & ० & o & 0.00 & 0.00 & 1.00 & 0.00 & 0.00 & 0.00 & 0.00 & 0.00 & 0.00 \\
\hline 235 & 3616.6 & $\begin{array}{l}\text { asym- } \\
\left(\mathrm{NH}_{2}\right)_{\mathrm{F}}\end{array}$ & 0 & O & O & O & 0.00 & 0.00 & 0.00 & 0.00 & 0.00 & 0.99 & 0.01 & 0.00 & 0.00 \\
\hline 236 & 3682.5 & $\mathrm{OH}_{\mathrm{S} 1-\mathrm{str}}$ & O & O & O & ० & 0.00 & 0.00 & 0.00 & 0.00 & 0.00 & 0.01 & 0.99 & 0.00 & 0.00 \\
\hline 237 & 3773.5 & $\mathrm{OH}_{\mathrm{S} 4-\mathrm{str}}$ & O & o & o & ○ & 0.00 & 0.00 & 0.00 & 0.00 & 0.00 & 0.00 & 0.00 & 1.00 & 0.00 \\
\hline
\end{tabular}

